\title{
A hyaluronic acid-based hydrogel enabling CD44-mediated chondrocyte binding and gapmer oligonucleotide release for modulation of gene expression in osteoarthritis
}

Yunpeng Cai ${ }^{1}$, Elena López-Ruiz ${ }^{1,2}$, Jesper Wengel ${ }^{3}$, Laura B. Creemers ${ }^{4}$ and Kenneth A. Howard $^{1, *}$

${ }^{1}$ The Interdisciplinary Nanoscience Center (iNANO), Department of Molecular Biology and Genetics, Aarhus University, 8000 Aarhus C, Denmark ${ }^{2}$ Department of Health Sciences, University of Jaén, Jaén E-23071, Spain. ${ }^{3}$ Nucleic Acid Center, Department of Physics, Chemistry and Pharmacy, University of Southern Denmark, 5230 Odense M, Denmark. ${ }^{4}$ Department of Orthopaedics, University Medical Center Utrecht, 3584 CX Utrecht, Netherlands

${ }^{*}$ Corresponding author at: Interdisciplinary Nanoscience Center (iNANO), 8000 Aarhus C, Denmark. E-mail address: kenh@inano.au.dk (K.A Howard).

Keywords: hyaluronic acid; hydrogel; osteoarthritis; CD44 receptor; gapmer; antisense 


\begin{abstract}
Hyaluronic acid (HA) is an attractive biomaterial for osteoarthritis (OA) treatment due to inherent functional and compatibility properties as an endogenous knee joint component. In this work, we describe a HA-based hydrogel with the dual functionality of increased CD44-dependent chondrocyte binding and controlled release of gapmer antisense oligonucleotides for unassisted cellular entry and subsequent gene silencing activity. A Schiff base-mediated gelation method was used to produce a panel of hydrogels varying in the aldehyde-modified HA (900 kDa) to chitosan ratios (3:7, 5:5 and 7:3) for identifying designs displaying optimal engagement of OA patient-derived CD44-expressing chondrocytes. Correlation was found between cell binding and CD44 expression, with maximal binding exhibited at a HA/chitosan ratio of 7:3, that was $181 \%$ higher than CD44-negative MCF-7 cell control cells. Transfection agent-free uptake into OA chondrocytes of fluorescent 13-mer DNA oligonucleotides with a flanked locked nucleic acid (LNA) gapmer design, in contrast to naked siRNA, was demonstrated by confocal and flow cytometric analysis. A sustained and complete release over 5 days was found with the 7:3 hydrogel, in contrast, the 5:5 and 3:7 hydrogel released $60 \%$ and $43 \%$ of loaded gapmers, respectively over the same period. A COX-2-specific gapmer designed with maximal chondrocyte gene silencing ( $70 \%$ silencing efficiency at $500 \mathrm{nM}$ compared with a mismatch gapmer sequence) resulted in effective COX-2 silencing over 14 days in hydrogels seeded with OA chondrocytes, with significant difference exhibited between day 3 and 10. This work introduces a novel HAbased CD44-mediated cellular binding and gapmer controlled release platform to modulate cellular gene expression.
\end{abstract}




\section{Introduction}

Osteoarthritis (OA) is a degenerative joint disorder that affects up to $50 \%$ of the US population over 50 years of age [1]. Currently no disease modifying treatments are available, and in end stage disease, arthroplasty is the only option. The synthetic activity of OA chondrocytes, which are the cellular components of the joint cartilage and considered to be terminally differentiated cells that maintain the extracellular matrix of the cartilage, is clearly increased but cannot counteract the ongoing catabolic processes [2].

Inflammation is thought to play a key role in the pain associated with OA and the induction of various extracellular matrix degrading enzymes that leads to the cartilage tissue degradation are linked with aberrant expression of inflammation-related target genes such as cyclooxygenase-2 (COX-2) [3, 4]. Maintaining the regenerative capacity of $\mathrm{OA}$ chondrocytes, whilst interrupting the production of inflammatory genes such as COX-2, is, therefore, an attractive therapeutic approach. Present anti-inflammatory treatments based on direct intra-articular injection of drugs such as corticosteroids only elicit short-term effects and non-specific in action $[5,6]$. In this work we propose a strategy based on a drug releasing platform able to interact and increase therapeutic delivery into chondrocytes.

RNA interference-based post-translational gene silencing with double stranded small interfering RNA (siRNA) offers the possibility of cytokine-specific reduction of activity, in contrast to global immunosuppression commonly associated with traditional anti-inflammatory drugs. The macromolecular size and polyanionic nature, however, requires delivery technologies such as cationic lipids or polymers to facilitate cellular entry, which necessitates careful design considerations to avoid toxicity [7]. Single stranded gapmer oligonucleotides are chimeric antisense oligonucleotides, containing a central block of deoxynucleotide monomers commonly flanked by a locked nucleic acid (LNA) sequence that interrupt mRNA expression by induction of RNase H activation [8, 9]. Significantly, the small size (11 13 nucleotides) allows unassisted cellular entry by gymnosis, without any requirement for a transfection agent. Moreover, fatty acid modifications of the gapmers have been shown to enhance the gene silencing efficiency, such as $\omega-6$ polyunsaturated fatty acids and oleic acids [10].

The use of hydrophilic polymeric hydrogel drug releasing platforms are an attractive approach in OA due to combined flexible mechanical properties that can serve as scaffold for tissue regeneration and partly mimic 
the cartilage. Hyaluronic acid (HA) is an endogenous synovial fluid component that lowers frictional forces in the knee joint that has recently been applied in OA research [11-13]. An unexplored HA property in OA treatment, however, is the ability to utilize the specific binding properties of HA for the CD44 receptor [14, 15] shown to be strongly expressed on bovine chondrocytes and normal human articular chondrocytes [16]. Previous work from our lab has demonstrated that the level of CD44 binding can be tuned by the HA molecular weight, with optimal engagement observed with high molecular weight HA [17] that offers a potential novel application using engineered HA hydrogel scaffolds for chondrocyte binding. This, combined with a capacity for sustained delivery of gapmers to inhibit COX-2 without the necessity of a transfection agent offers a novel dual functional platform for cartilage repair and interruption of damage progression.

In this work oxidized high molecular weight HA (900 kDa) was cross-linked with chitosan to form an in situ hydrogel by a Schiff reaction for CD44-mediated chondrocytes binding studies using CD44-expressing human primary chondrocytes and isogenic MCF-7 CD44-expressing and CD44-negative cells. Optimal COXdesigns identified in an in vitro screen were then loaded into the hydrogel to study the release profiles and subsequent anti-inflammation effects in IL-1 $\beta$ induced OA chondrocytes over a 2-week period. 


\section{Materials and Methods}

\subsection{Materials}

Chitosan (degree of deacetylation $>90 \%$, Mw 200-600 kDa) was purchased from Novamatrix, Sandvika, Norway. Hyaluronic acid (HA) (Mw $900 \mathrm{kDa}$ ) was purchased from Lifecore biomedical, Chaska, USA, CyQuant cell proliferation kit from ThermoFisher Scientific, Waltham, USA and sodium periodate, t-butyl carbazate, $\beta$-glycerol phosphate disodium salt, ethylene glycerol, hyaluronidase and anti-CD44 FITC antibody from Sigma-Aldrich. Ultrapure water (Siemens Ultra Clear Basic plus) was used in all experiments.

COX-2 antisense oligonucleotides (ASOs) gapmer sequences used: Gapmer design 1: 5'-lGlGlAlT dGdCdCdAdGdTdGdAdTdA 1GlAlGlG-3', Gapmer design 2: 5'-1GlGlAlT dGdCdCdAdGdTdGdA lTlAlGlA-3', palmitoylated gapmer design 3: 5'-1GlGlAaT dGdCdCdAdGdTdGdA aTlAlGlA-3', Gapmer design 4: 5'-1GlGlA dAdAdCdAdTdCdGdAdC 1AlGIT-3'. Cy5 labelled gapmer: 5'-1GlGIAlT dGdCdCdAdGdTdTdA 1GlAlGlG-3', Cy5 labelled palmitoylated gapmer: 5'-1GlGlA aTdGdCdCdAdGdTdGdAaT 1AlGlA, FAM labelled gapmer: 5'-1GlGlA dAdAdCdAdTdCdGdAdC 1AlGlT3' (1 - locked nucleic acid, d-deoxyribonucleotide, a - palmitoylated amino locked nucleic acid). All ASO were synthesized as all-phosphorothioate linked sequences except for the Cy5 labelled palmitoylated gapmer which was synthesized as all-phosphordiester linked sequence. ASOs were dissolved in nuclease-free water (Ambion, Austin, TX) to $200 \mu \mathrm{M}$ concentrations measured by an Implen NanoDrop (Thermo Scientific, and stored at $-20^{\circ} \mathrm{C}$. siRNA was purchased from Genepharma (Shanghai, China) with the following sequences for the COX-2 siRNA-sense strand: 5'-CAUUCCCUUCCUUCGAAAUdTdT-3' and antisense strand: 5'AUUUCGAAGGAAGGGAAUGdTdT-3'. siRNA was diluted using nuclease-free water. The phosphoramidite building block used to incorporate the palmitoylated amino locked nucleic acid thymine monomer is commercially available from RiboTask ApS (www.ribotask.com).

Primary chondrocytes were isolated from articular cartilage of OA patients undergoing total knee arthroplasty. A CD44-negative MCF-7 cell line was derived from the original MCF-7 cell line (obtained from ATCC) via subcloning after stable insertion of a sequence suitable for subsequent Flp-recombinase-mediated insertion of expression plasmids. The cell line was used to insert the coding sequence (CDS) of the standard 
variant of CD44, known as CD44S or CD44 isoform 4 (NM_001001391.1; Consensus CDS CCDS31457.1) under the control of a constitutive cytomegalovirus promoter, which yielded the CD44-expressing cells [17].

\subsection{Synthesis of aldehyde modified hyaluronic acid}

Aldehyde-HA was synthesized by an oxidization method described previously [13]. Briefly, $1.0 \mathrm{~g}$ HA ( $2.5 \mathrm{mmol}$ ) was dissolved in $100 \mathrm{~mL}$ deionized water at a concentration of $10 \mathrm{mg} / \mathrm{ml} .0 .54 \mathrm{~g}$ sodium periodate (2.5 mmol) was dissolved in $5 \mathrm{ml}$ deionized water and added dropwise into the HA solution under stirring in the dark. After $2 \mathrm{~h}, 1 \mathrm{ml}$ of ethylene glycol was added into the reaction system to neutralize the excess amount of sodium periodate, and stirred for a further $1 \mathrm{~h}$. The aldehyde-HA product was then dialyzed for 3 days against deionized water (changed daily) and then freeze-dried. The oxidation degree of HA was determined by a t-butyl carbazate method described previously [18].

\subsection{Preparation of hyaluronic acid/chitosan hydrogels}

$200 \mathrm{mg}$ chitosan was dissolved in $9 \mathrm{ml} 0.1 \mathrm{M} \mathrm{HCl}$ solution, and then $1 \mathrm{ml}$ of $560 \mathrm{mg} \beta$-glycerol phosphate solution added dropwise on ice, resulting in a chitosan solution at neutral $\mathrm{pH}$ [19]. Aldehyde-HA (HA) was dissolved in deionized water at a concentration of $20 \mathrm{mg} / \mathrm{ml}$. Both the HA solution and chitosan solution were sterilized by UV irradiation before mixing. The hydrogel was prepared by mixing HA and chitosan solutions at different volume ratios (3:7, 5:5 and 7:3) and gelation initiated spontaneously upon mixing. HA solution and chitosan solution were mixed in 24 -well plate wells in a total volume of $500 \mu$ land incubated for 10 minutes to enable complete gelation. For gapmer incorporation, 4 nmol gapmers were first mixed with the HA solution, after which the HA/gapmer solution was mixed with the chitosan solution on ice.

\subsection{Determination of CD44 expression in primary chondrocytes and isogenic MCF-7 cells}

All cells were cultured at $37^{\circ} \mathrm{C}$ under $5 \% \mathrm{CO}_{2}$ in a water saturated atmosphere. For chondrocyte culture, Dulbecco's Modified Eagle's Medium (DMEM D6429) with $1 \%$ Penicillin-Streptomycin and $10 \%$ heatinactivate fetal bovine serum was used. MCF-7 (passage 2-8) media was composed of DMEM Glutamax 
medium supplemented with fetal bovine serum (10\%), Streptomycin/Penicillin (1\%), insulin $\left(10 \mu \mathrm{g} \mathrm{ml}^{-1}\right)$, and from the fourth day post plating the MCF-7, media was supplemented with hygromycin $\left(120 \mu \mathrm{g} \mathrm{ml}^{-1}\right)$.

OA Chondrocytes and isogenic MCF-7 cells (CD44-expressing and CD44-negative) were seeded at $1 \mathrm{x}$ $10^{5}$ cells/well in a 12-well plate. The following day, cells were trypsinized and incubated with 5 ul Anti-CD44 FITC antibody in $100 \mu \mathrm{l}$ blocking buffer ((3\% BSA in phosphate buffered saline (PBS) solution)) for 30 mins. After 3 times wash with blocking buffer, the cells were resuspended in PBS and the fluorescence intensity determined by flow cytometry using a Gallios (Beckman Coulter) flow cytometer with a $488 \mathrm{~nm}$ laser. The cell population gate was based on forward and side scattering, and was gated to distinguish dead cells and cell doublets. Events was set to 10000 for each measurement and samples were in triplicate.

\subsection{Cellular binding to the hyaluronic acid-based hydrogel}

OA chondrocytes, CD44-expressing and negative MCF-7 cells were selected to compare the CD44dependent binding of the HA-based hydrogel. HA and chitosan solutions of different ratios (3:7, 5:5 and 7:3) were pipetted into a 12 -well plate to form hydrogels and $5 \times 10^{4}$ cells were then seeded on top in $0.5 \mathrm{ml}$ media. After $36 \mathrm{~h}$ incubation, the media was changed to remove unattached cells, and fresh medium containing 100 $\mathrm{U} / \mathrm{ml}$ hyaluronidase was then added and incubated for $5 \mathrm{~h}$ for hydrogel digestion. The supernatants containing cells attached to the hydrogel were collected and the wells (with remaining attached cells) were treated with $200 \mathrm{ul}$ trypsin for $5 \mathrm{~min}$. The mixtures of supernatant and trypsin were then centrifuged and lysed for CyQuant cell proliferation assay. All the cell numbers of different cell types were normalized to the numbers of corresponding cells plated in wells to eliminate the difference in proliferation rates.

A 3-(4, 5-dimethylthiazol-2-y1)-2, 5-diphenyltetrazolium bromide (MTT) assay was used to determine cell viability of chondrocytes attached to the hydrogels. Collected chondrocytes pellets from the hydrogels were treated with $200 \mu \mathrm{MTT}(5 \mathrm{mg} / \mathrm{ml})$, and incubated for $3 \mathrm{~h}$ at $37^{\circ} \mathrm{C}$. Formazan crystals were then dissolved in $150 \mu \mathrm{l}$ of dimethyl sulfoxide (DMSO). The absorbance at $570 \mathrm{~nm}$ was measured with a Victor X5Multilabel plate reader (PerkinElmer) and live cell numbers were calculated by a calibration curve from a non-treated group. Total cell numbers were determined using a NucleoCounter® NC-200 (ChemoMetec) and viabilities were calculated as percentage of live cells/total cells. 
2.6 Cellular uptake of gapmer oligonucleotides determined by flow cytometric and confocal microscopic analysis

$1 \times 10^{5} \mathrm{OA}$ chondrocytes were seeded in a 24 -well plate. After $24 \mathrm{~h}, 50 \mathrm{nM}$ or $100 \mathrm{nM}$ Cy5 labelled gapmers (non-modified gapmer: 5'-1GlGlAlT dGdCdCdAdGdTdTdA IGlAlGlG-3', palmitoylated gapmer: 5'lGlGlA aTdGdCdCdAdGdTdGdAaT 1AlGlA) and $50 \mathrm{nM}$ naked siRNA were added to the media. The media was removed after $24 \mathrm{~h}$ and chondrocytes were washed 3 times with PBS. The chondrocytes were then trypsinized, and the fluorescence intensity determined by flow cytometry using a $633 \mathrm{~nm}$ laser.

For confocal microscopic studies, $1 \times 10^{5} \mathrm{OA}$ chondrocytes were seeded in a 24 -well plate. After $24 \mathrm{~h}$, $50 \mathrm{nM}$ or $100 \mathrm{nM}$ FAM labelled non-modified gapmer (5'-1GlGlA dAdAdCdAdTdCdGdAdC 1AlGIT-3') were added to the media. The media was removed after $24 \mathrm{~h}$ and the cells were washed twice with PBS, harvested by tripsinization and fixed with $4 \%$ paraformaldehyde for $15 \mathrm{~min}$, permeabilized and stained with Hoechst 33342 and phalloidin (Life technologies) before confocal microscopic analysis using a LSM700 (Zeiss).

\subsection{COX-2 cellular gene silencing in vitro}

OA chondrocytes were seeded at $1 \times 10^{5}$ in a 6 -well plate and $10 \mathrm{ng} / \mathrm{ml} \mathrm{IL}-1 \beta$ added for induction of inflammation at day 0 and day 7. After $24 \mathrm{~h}, 500 \mathrm{nM}$ naked COX-2 gapmer (4 designs) or $50 \mathrm{nM} \mathrm{COX-2}$ siRNA (antisense strand: 5'-AUUUCGAAGGAAGGGAAUGdTdT-3') with $4 \mu$ l lipofectamine were added into the media and incubated for another two days before RNA extraction. The COX-2 mRNA expression level was quantified by real time polymerase chain reaction (PCR), normalized to Glyceraldehyde 3-phosphate dehydrogenase (GAPDH). Thermal cycling conditions were $48{ }^{\circ} \mathrm{C}$ for $15 \mathrm{~min}$ of activation, $95{ }^{\circ} \mathrm{C}$ for $10 \mathrm{~min}$ of amplification and 40 cycles of $95{ }^{\circ} \mathrm{C}$ at $15 \mathrm{~s}$ denaturing and $60{ }^{\circ} \mathrm{C}$ at $60 \mathrm{~s}$ annealing/extending. COX-2 forward primer: 5'-TGGCTACAAAAGCTGGGGAAG-3', reverse primer: 5'AACTGATGCGTGAAGTGCTG-3'. GAPDH forward primer: 5'-GTCAGCCGCATCTTCTTTTG-3', reverse primer: 5'-GCGCCCAATACGACCAAATC-3'. 
For cell viability evaluation, chondrocytes were seeded in a 48 -well plate at a density of $1 \times 10^{4} /$ well. The cells were treated with $500 \mathrm{nM}$ gapmers or $50 \mathrm{nM}$ siRNA with $4 \mu \mathrm{llipofectamine}$ for $24 \mathrm{~h}$. Subsequently, MTT assay was performed as described in section 2.5 and viabilities were normalized to a non-treated group.

2.8 Gapmer oligonucleotide release profiles and in vitro COX-2 inhibition after inclusion within a hyaluronic acid-based hydrogel

Hydrogels of different HA: chitosan ratios (3:7, 5:5 and 7:3) were prepared to modulate the gapmer release profiles. $50 \mu \mathrm{g}$ Cy5 labelled gapmer (7.5 nmol, 5'-1GlGlAlT dGdCdCdAdGdTdTdA 1GlAlGlG-3') was added into the HA solution and mixed with chitosan solution in a 12-well plate to form hydrogel at a total volume of $2 \mathrm{ml}$, and the hydrogels were then incubated in $1 \mathrm{ml} \mathrm{PBS}$. At different time points over a 5 day period, $1 \mathrm{ml}$ of PBS was removed and the fluorescence intensity determined by a Victor X5Multilabel plate reader (PerkinElmer). The hydrogel incubation buffer was replenished with $1 \mathrm{ml}$ fresh PBS after each reading. At day 6, $100 \mathrm{U} / \mathrm{ml}$ hyaluronidase was added to $1 \mathrm{ml} \mathrm{PBS}$ in order to digest the hydrogel and the supernatants collected for fluorescence analysis. A calibration curve of the same Cy5 gapmers was made to quantify the amount of released gapmer. Loading efficiency of gapmers in hydrogels were determined by a depletion method, as total released gapmers and gapmers in digested solutions were summed up to be total encapsulation amount.

The capability for hydrogel released gapmers to reduce COX-2 expression in OA chondrocytes was investigated $1 \times 10^{5} \mathrm{OA}$ chondrocytes were seeded in 6-well plates, and $10 \mathrm{ng} / \mathrm{ml} \mathrm{IL-}$ $1 \beta$ was added into the medium to induce inflammation at day 0 and day 7 . Two mls of the hydrogel (HA: chitosan 7:3) containing 4 nmol anti-COX-2 gapmer design 1 (5'-1GlGlAlT dGdCdCdAdGdTdGdAdTdA lGlAlGlG-3') was pipetted into the wells to overlay the seeded cells, and the media changed every 3 days. A mismatch gapmer group and IL-1 $\beta$ free group were used as controls. At different time points, total RNA was extracted and COX-2 mRNA expression levels were determined by PCR as in the above section 2.7. 


\subsection{Statistical analysis}

A one-way ANOVA was used for cellular binding and COX-2 inhibition experiments with each experiment performed twice. The data is presented as means \pm standard error of the mean. Differences were considered to be significant at a $\mathrm{p}$ value of 0.05 . 


\section{Results}

A panel of hydrogels was constructed from different ratios of chitosan and aldehyde modified HA introduced to HA by oxidation of sodium periodate. The vicinal hydroxyl groups were oxidized to dialdehydes by opening the sugar ring. The mole ratio between HA monomer and oxidant was $1: 1$, with $\sim 38.6 \%$ of the hydroxyl groups oxidized determined by a t-butyl carbazate method.

$\beta$-glycerol phosphate was added to the chitosan in $0.1 \mathrm{M} \mathrm{HCl}$ to neutralize the acidic solution and maintain the chitosan in soluble form as a result of glycerol moiety separating the chitosan chains to maintain solubility [20]. After the addition of $560 \mathrm{mg} \beta$-glycerol phosphate solution, the $\mathrm{pH}$ increased from 3.9 to 7.0 , which was close to physiological $\mathrm{pH}$ value. The hydrogel gelated on mixing the chitosan with the HA solution due to a Schiff base reaction, with the HA aldehyde groups cross-linking with carboxyl groups on chitosan.

The fluorescent intensity of an anti-human CD44 fluorescent antibody using flow cytometry was used to determine the CD44 expression level on the different cell types. CD44-negative MCF-7 cells did not show any antibody binding but the CD44-expressing MCF-7 showed CD44 expression (Supplementary Figure 1). A 5.6fold higher expression of CD44 was found in primary chondrocytes compared to the CD44-expressing MCF7 cell line that supports the approach for CD44-dependent chondrocyte binding into an HA hydrogel matrix. This was investigated with the panel of cells seeded onto hydrogels ranging in HA content. Overall chondrocyte viability was around $75-80 \%$ after $36 \mathrm{~h}$ incubation on the hydrogels (Supplementary Figure 2). All the cell numbers of different cell types were normalized to the numbers of corresponding cells plated in wells to eliminate difference of cell proliferation rates. Significant differences in binding was found for OA chondrocytes than the CD44-expressing and CD44-negative MCF-7 cells (Figure 1), with higher binding found with the higher CD44 expression. In the HA/chitosan 7:3 hydrogel, the chondrocyte normalized number was $181 \%$ higher than CD44-negative MCF-7 cells and $100 \%$ higher than low CD44-expressing MCF-7 cells, respectively. In the HA/chitosan 5:5 hydrogel, normalized chondrocytes number was 200\% higher than CD44negative MCF-7 cell, but normalized total chondrocyte number decreased from 1.19 to 0.79 by $34 \%$ compared with 7:3 group. In the HA/chitosan 3:7 hydrogel, no significant difference of cell numbers was found between the cells differing in CD44 expression, which supports the cell number differences were attributed to the dependency on the CD44-HA interaction. 


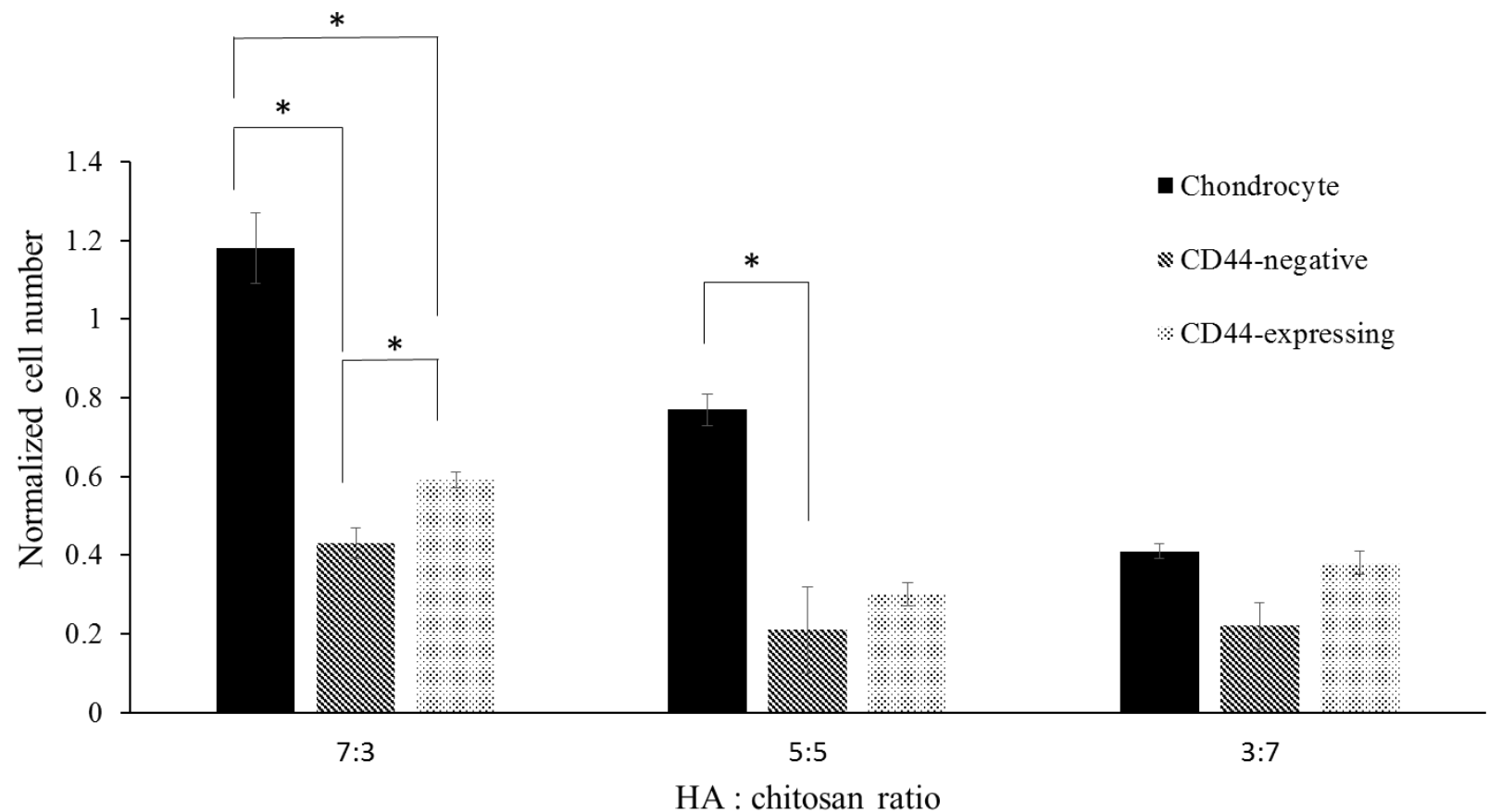

Fig. 1. Cellular binding to hydrogels composed of different HA: chitosan ratios (7:3, 5:5 and 3:7). Chondrocytes, CD44-expressing and CD44-negative MCF-7 cells were seeded on hydrogels and incubated for 36 h. After $3 \times$ PBS wash, binding cell numbers were determined and normalized to attached cell numbers in plate wells $(N=3)$. Asterisk denotes statistical significance.

The ability of fluorescent labelled gapmer oligonucleotides to associate and enter OA chondrocytes without the requirement of a transfection agent was validated by flow cytometry and confocal microscopy (Figure 2). Non-modified gapmers showed a 5-fold higher association compared with palmitoylated modified, whilst naked siRNA and non-treated group showed no fluorescent signals (Figure 2). Treatment of $100 \mathrm{nM}$ gapmers showed a higher cellular association with OA chondrocytes than $50 \mathrm{nM}, 1.8$-fold increase for nonmodified gapmer and 1.75-fold increase for palmitoylated gapmer respectively. OA chondrocytes incubated for $24 \mathrm{~h}$ with either 50 or $100 \mathrm{nM}$ gapmer showed green fluorescence throughout the cytoplasm that was absent from the nucleus (Figure 2). 


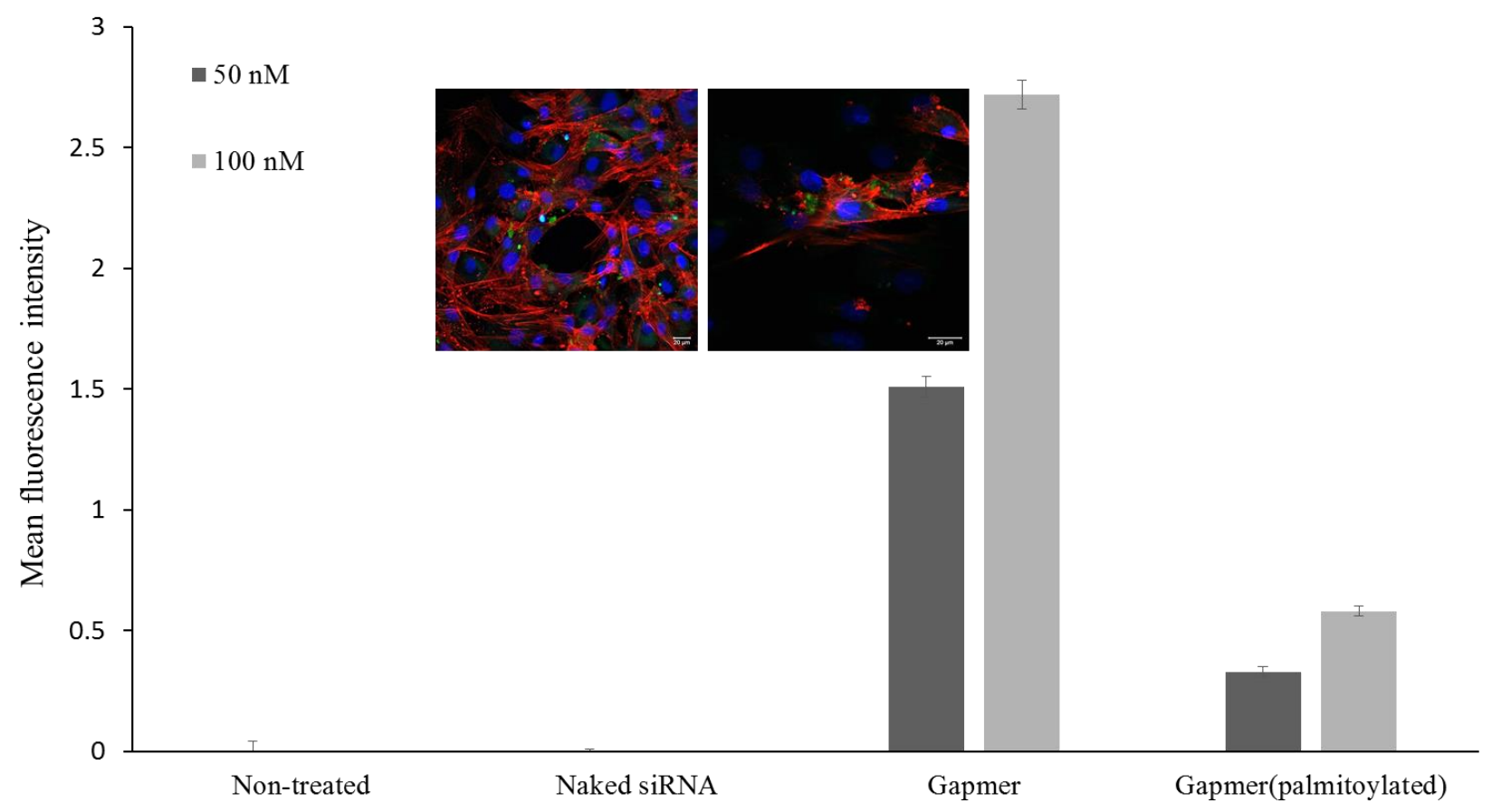

Fig. 2. Cellular uptake of fluorescence-labelled gapmer oligonucleotides in primary OA chondrocytes. Cy5 labelled gapmers (50 and $100 \mathrm{nM}$ ), with and without palmitoylation, were incubated with primary OA chondrocytes for $24 \mathrm{~h}$, and fluorescence intensity determined using flow cytometry. Naked Cy5 labelled siRNA at a concentration of $50 \mathrm{nM}$ was used as a negative control. Flow cytometer was determined with a laser wavelength of $633 \mathrm{~nm}$ and gate events of $10000(N=3)$. Insert: Left panel shows primary OA chondrocytes with $50 \mathrm{nM}$ gapmer without palmitoylation, and right panel shows primary OA chondrocytes with $100 \mathrm{nM}$ gapmer without palmitoylation. Nuclei were stained with Hoechst (blue), actin filaments were stained with phalloidin (red) and gapmer were labelled with FAM (green). Original magnification 20x.

A panel of COX-2 specific gapmer designs of different sequences, with or without palmitoylation, were screened in OA chondrocytes for unassisted (without transfection agent) gene silencing compared to a COX2 siRNA with lipofectamine transfection (Figure 3). Successful silencing of target gene COX-2 ( 82\% silencing efficiency) was seen in vitro for the lipofectamine-mediated siRNA positive control. The silencing efficiency of different gapmer sequences were different dependent on design with gapmer design 1 (5'1GlGlAlT dGdCdCdAdGdTdGdAdTdA IGlAlGlG-3') exhibited the highest silencing efficiency of $70 \%$ and 
gapmer design 3 was $33 \%$. A 10-fold higher concentration was required for unassisted gapmer silencing compared with lipofectamine-mediated siRNA. Gapmer design 1 showed a better knockdown compared to palmitoylated gapmer design 3 mainly attributed to different nucleotides in sequences. It should be noted that exchange of two LNA nucleotides of gapmer design 2 with two palmitoylated amino-LNA nucleotides to give gapmer design 3, makesan inactive ASO into an active one (gapmer design 3). Moreover, the four gapmers displayed a higher chondrocyte viability of $85-95 \%(92.3 \%, 94.2 \%, 88.5$ and $84.6 \%$ respectively) compared with siRNA (73\%) transfected with lipofectamine evaluated by an MTT assay (Supplementary Figure 3). Gapmer design 1 was used for subsequent experiments based on the in vitro cellular gene silencing screen.

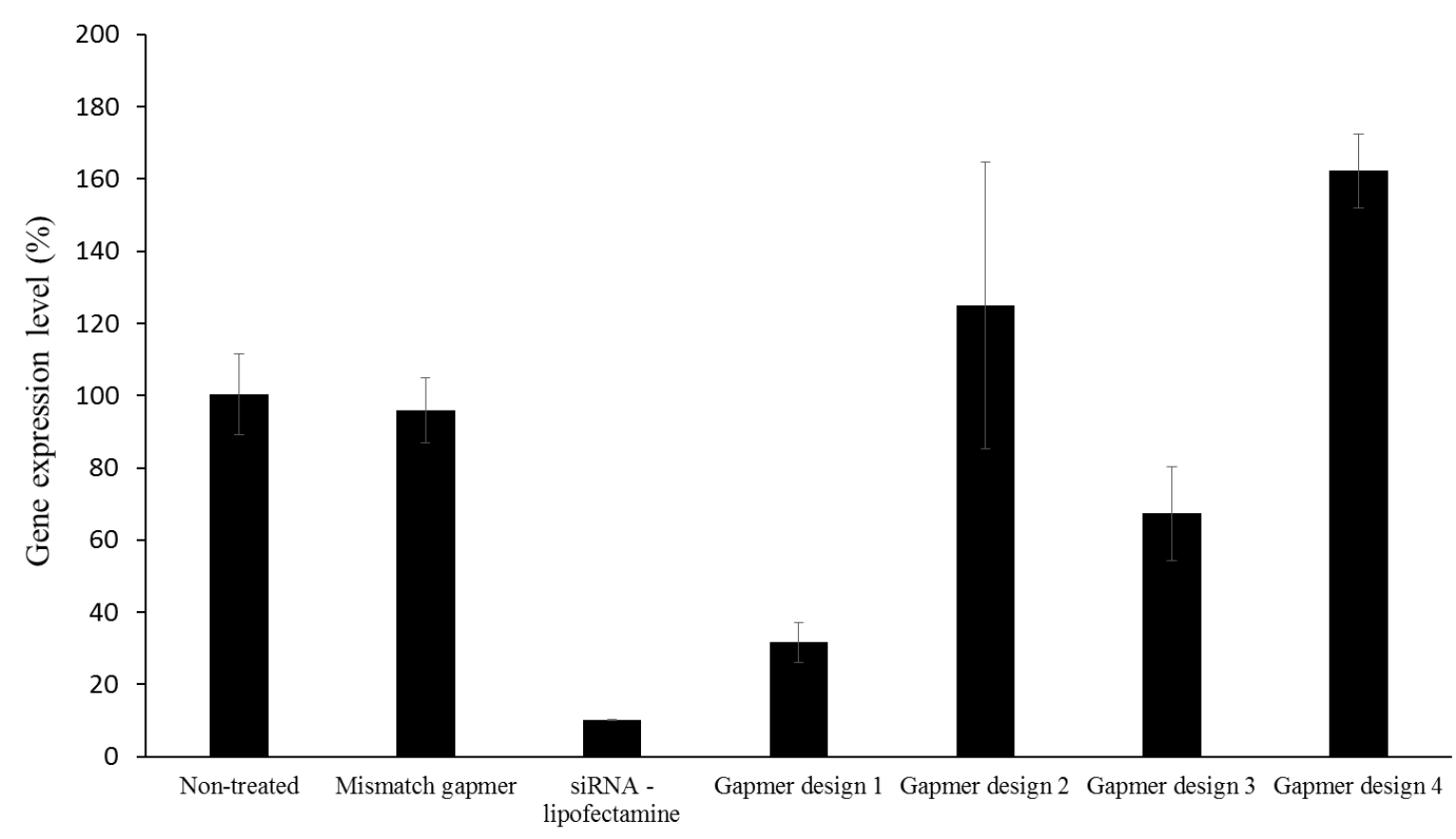

Fig. 3. COX-2 cellular gene in vitro using different gapmer oligonucleotide designs in primary $O A$ chondrocytes. Primary OA chondrocytes were treated with $500 \mathrm{nM}$ gapmers of different designs. After $24 \mathrm{~h}$ incubation, $10 \mathrm{ng} / \mathrm{ml} \mathrm{IL}-1 \beta$ was added to induce inflammation. After 4 days incubation, the RNA was extracted for real time PCR analysis to determine COX-2 gene expression levels. COX-2 siRNA with lipofectamine 2000 was used as a positive control at a concentration of $50 \mathrm{nM}$. (N=3) Gapmer design 1: 5'-lGlGlAlT dGdCdCdAdGdTdGdAdTdA lGlAlGlG-3', Gapmer 2 design: 5'-lGlGlAlT dGdCdCdAdGdTdGdA lTlAlGlA3', Gapmer 3 design: 5'-lGlGlAaT dGdCdCdAdGdTdGdA aTlAlGlA-3', Gapmer design 4: 5'-lGlGlA dAdAdCdAdTdCdGdAdC lAlGlT-3'. (l-locked nucleic acid, $d$-deoxyribonucleotide, a-palmitoylated amino 
locked nucleic acid). COX-2 siRNA-sense strand: 5'-CAUUCCCUUCCUUCGAAAUdTdT-3' and antisense strand: 5'-AUUUCGAAGGAAGGGAAUGdTdT-3'.

Hydrogels at different HA: chitosan ratios were prepared to modulate the release profile of fluorescent gapmers measured by a fluorescent plate reader. Incorporation efficiency was $96.1-97.2 \%$, with highest incorporation efficiency achieved with the hydrogel of HA/chitosan ratio of 7:3. A 5-day sustained release profile was shown with the HA-chitosan 7:3 hydrogel, while 5:5 hydrogel released over 3 days and 3:7 hydrogel within 1 day (Figure 4). For the HA:chitosan 7:3 hydrogel, the total amount released was 101\%, and for the 5:5 and the 3:7 hydrogels $60 \%$ and $43 \%$, respectively. The initial burst in first 4 hours for the 7:3 hydrogel was $41 \%$, whilst 5:5 and 3: 7 hydrogels were $56 \%$ and $62 \%$, respectively. No loss of structural integrity was observed after release over the 5 day period demonstrated by non-detection of fragment bands (Supplementary Figure 4). The fainter band at day 5 can be attributed to the low amount released between day 4 and day 5 .

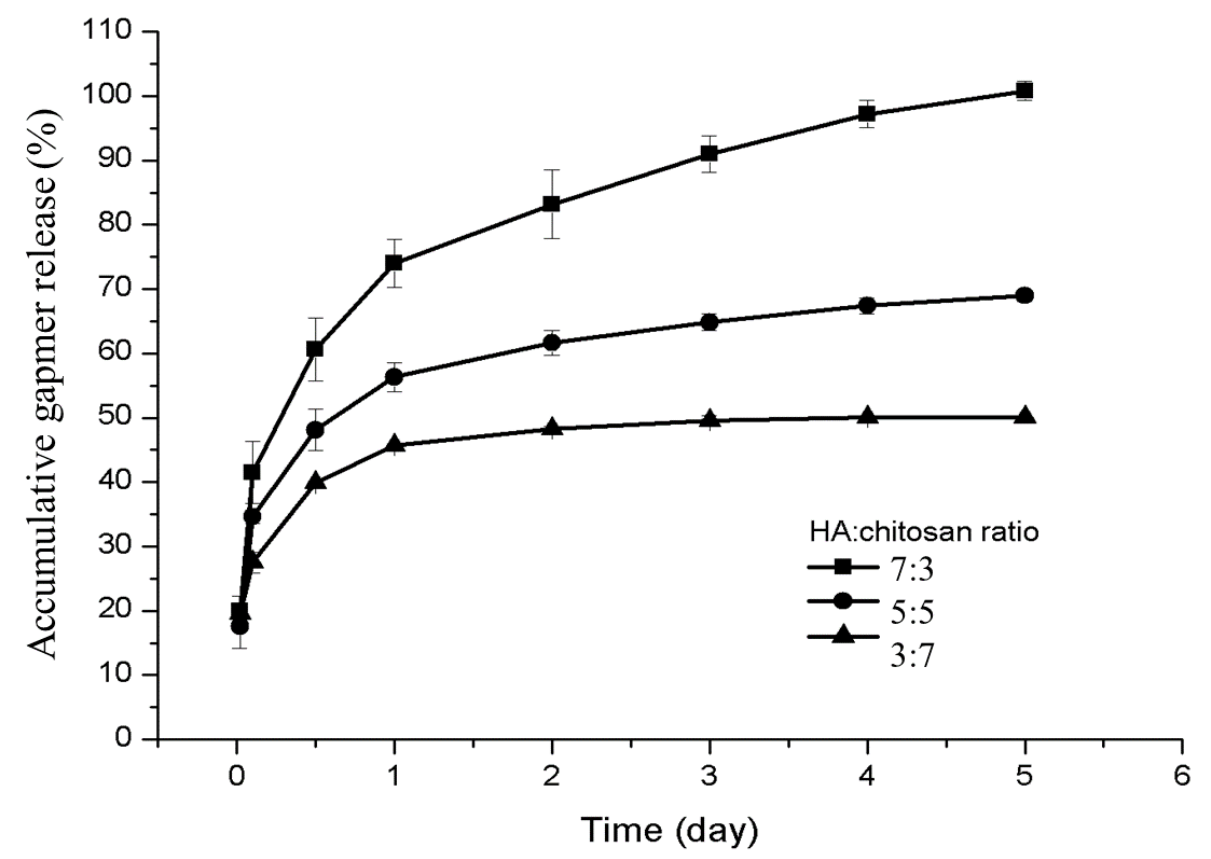

Fig. 4. Accumulative release profiles of gapmer oligonucleotides in different hydrogel compositions. Cy5 labelled gapmer (50 ug) was incorporated into hydrogels composed of different HA:chitosan ratios (7:3, 5:5 
and 3:7). $2 \mathrm{ml}$ hydrogel was added into a 24-well plate and $0.5 \mathrm{ml} \mathrm{PBS}$ was used as release media.

Fluorescence intensity of media was determined at an excitation wavelength of $650 \mathrm{~nm}$ and emission

wavelength of $670 \mathrm{~nm}$. Released gapmer amount was calculated using a standard calibration curve. $(N=3)$.

A hydrogel containing a COX-2 specific gapmer design 1 exhibited a sustained COX-2 inhibition over 14 days. IL-1 $\beta$ was used to induce inflammation in primary OA chondrocytes and increased the COX-2 level more than 100-fold compared with non-treated cells (Figure 5). A second IL-1 $\beta$ administration to OA chondrocytes at day 7 was performed to compensate for the loss of IL-1 $\beta$, asCOX-2 level decreased to $49 \%$ at day 6 compared to day 1. From day 3, a significant reduction of COX-2 expression level was observed for the COX-2 gapmer treated cells, with silencing maintained up to day 10. At day 14, the COX-2 specific gapmer group COX-2 expression level increased to a similar level to the mismatch control group, most probably due to the completion of the COX-2 inhibition effect of the released gapmers.

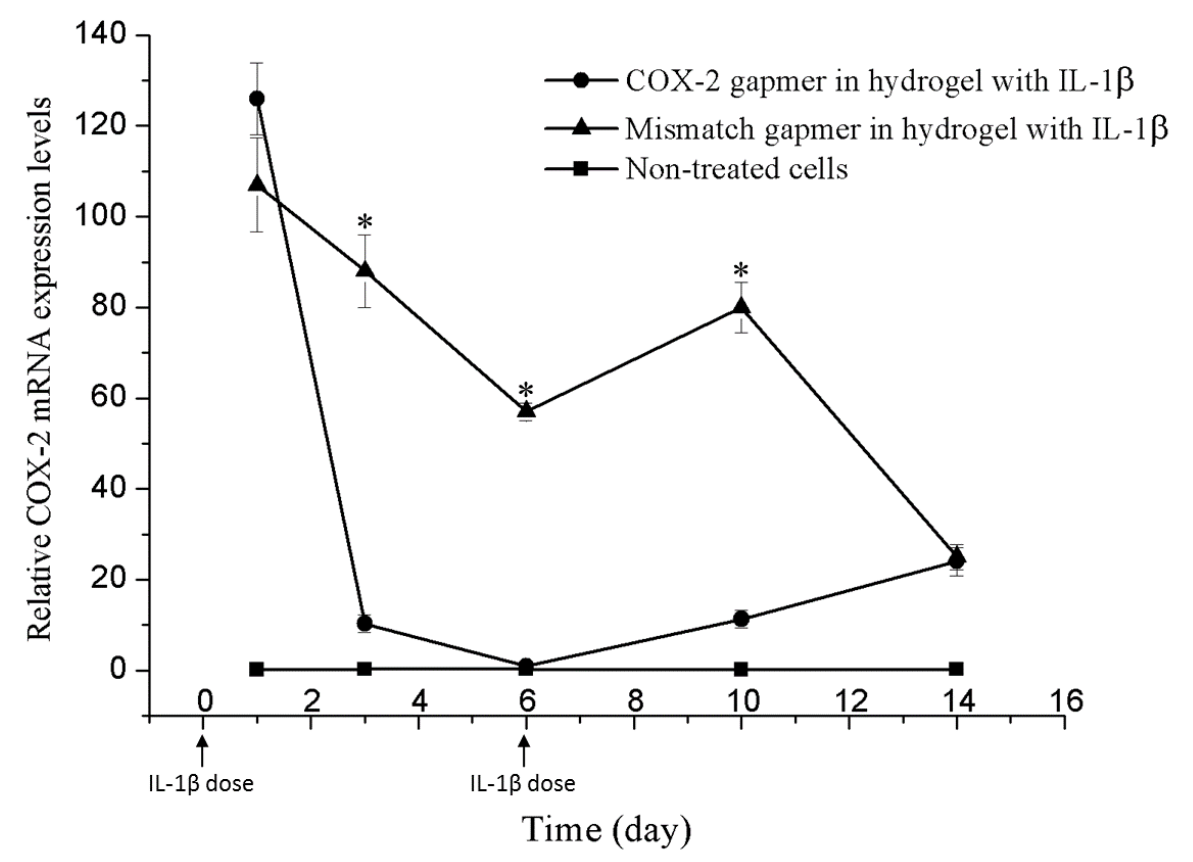

Fig. 5. COX-2 cellular gene expression level from 1 to 14 days in primary OA chondrocytes below gapmer oligonucleotide loaded hydrogels. COX-2 gapmers were incorporated in a HA-based hydrogel (HA:chitosan of 7:3). Primary OA chondrocytes were treated with $10 \mathrm{ng} / \mathrm{ml} \mathrm{IL}-1 \beta$ to induce inflammation at day 0 and 7 (arrows). At different time points, RNA was extracted for real time PCR analysis. COX-2 expression levels 
were normalized to non-treated groups. A hydrogel loaded with a mismatch gapmer sequence was used as negative control. Asterisk denotes significant differences between experimental and negative control groups $(N=3)$. 


\section{Discussion}

In this work, we introduce a novel HA-based hydrogel designed for the dual capacity for CD44-dependent chondrocyte binding and gene regulation for applications in the treatment of OA. The dual modality of chondrocytes engagement and simultaneous sustained release of gapmers for unassisted cell entry, potentiates interaction between drugs and cells required for enhanced tissue regeneration within a biodegradable 3dimensional scaffold.

Current use of HA in OA treatment is mainly focused on chondroprotection, anti-inflammatory and friction reduction [21, 22]. HA-based hydrogels such as HA-fibrin [12], HA-chitosan [23] and methacrylated HA [24] hydrogels have been used for OA due to mechanical and biocompatibility properties as a natural component in knee joint. Typically, high molecular weight HA has been used to facilitate a more stable hydrogel matrix structure. There is also evidence showing the size of HA molecules and concentration are lower in OA patients with chronic or acute inflammation, and that the supply of high molecular weight HA can both stimulate the HA synthesis in synovium, and mediate chondrocyte proliferation and function [25].

To our knowledge no previous studies have utilised HA-based hydrogels for active CD44-mediated binding of chondrocytes as an approach for OA tissue repair, which was based on our previous finding of higher CD44 engagement with high $900 \mathrm{kDa}$ molecular weight HA [17]. Although it is termed as 'binding' in this work, and cell movement into hydrogel was not investigated in the present study, CD44 interactions may lead to active recruitment of chondrocytes into the porous network of hydrogel. A previous study has reported the use of hydrogel immobilized CD34 antibody to capture endothelial progenitor cells by utilizing receptor binding [26]. Incorporation of labile biomacromolecules such as antibodies as a method for cell binding or recruitment, however, is restricted by the necessity for conjugation and stability. The preferential binding of surrounding cells into a drug-containing 3-D matrix offers great potential in tissue regeneration applications as an alternative to loading patient-specific chondrocytes into the hydrogels before implantation that requires patient cell harvest. Previous reports have suggested the role of HA in the recruitment of CD44-expressing cancer cells in endogenous extracellular matrix possibly due to HA presence $[27,28]$. However, there are no reports on CD44-expressing chondrocyte binding to HA-rich matrix and the application for OA treatment. 
In our work the HA was modified for introduction of aldehyde groups to allow cross-linking with the carboxyl-bearing chitosan. The gelation process took $\sim 2$ minutes, suggesting potential use as an injectable soluble formulation in the joint for in situ gelation. An important consideration was to retain the HA conformation that allows effective interaction with CD44 that has previously been reported to be dependent on three carboxyl groups [29]. For instance, a $50 \mathrm{~mol} \%$ HA modification of adipic acid dihyrazide has been shown to not bind CD44 [30]. In our work, a higher primary chondrocyte number, compared to the CD44expressing and CD44-negative cell line, was shown with increased $900 \mathrm{kDa} H A$ content that is most probably attributed to the higher CD44-expression level determined in these cells. The isogenic MCF-7 cells were used as models to study HA-CD44 specific binding, so differences could be attributed to CD44 expression level only, with the CD44-expressing cells, in contrast to the CD44-negative MCF-7 cells, exhibiting a higher binding with a HA-rich matrix.

The hydrogel was used to incorporate the polyanionic gapmers of $\sim 6 \mathrm{kDa}$ molecular weight. To our knowledge, this is the first demonstration of incorporation and sustained release of gapmers from a HA-based 3-D scaffold. The hydrogel is a highly water-containing matrix that allows loaded small hydrophilic molecules to diffuse into the outer aqueous environment at a relatively rapid speed [31]. It is, therefore, unlikely that gapmers were physically entrapped within the matrix, but rather incorporated by electrostatic interactions between the phosphate backbone of the gapmer and the amino-bearing chitosan, that result in a high encapsulation efficiency of $\sim 96.1-97.2 \%$. The release of gapmers can be attributed to the exchange of polyanions in the culture medium, which may occur in an in vivo environment due to anionic peptides or chloride ions in body fluids. The hydrogel with the highest HA percentage of $70 \%$ displayed a more complete release and lower initial burst than the other compositions. This could be attributed to the tighter binding of the gapmers with higher chitosan contents, restricting release prior to hydrogel degradation, which may take more than 1 month [32]. The HA: chitosan 7:3 hydrogel was selected for subsequent silencing studies due to its favorable gapmer release profile and high chondrocyte binding.

The attractive properties of gapmers are specificity, stability and ability for unassisted cell entry by the process of gymnosis to facilitate gene silencing. Gymnosis is the process of the cellular delivery of antisense oligodeoxynucleotides to cells, in the absence of any carriers or conjugation, that produces sequence-specific 
gene silencing [10]. This abrogates transfection agent inclusion and makes gapmers suitable for sustained release and subsequent entry into surrounding cells. Several gapmer designs were screened to select designs showing maximal COX-2 cellular gene silencing for downstream hydrogel studies. Palmitoylated gapmers were expected to have higher cell uptake due to the hydrophobic property of fatty acid that can bind to the cell membrane via hydrophobic interaction. However, it showed less uptake and silencing efficiency compared with the optimal non-modified gapmer. This could, among other factors (the insertion of different numbers of LNAs or different nucleotides in designs), be due to it being a saturated fatty acid derivative, in contrast to unsaturated fatty acid shown previously to enhance gene silencing [10].

$\mathrm{COX}-2$ is one of the main inflammatory targets in OA, and IL-1 $\beta$ has been widely used to induce inflammation in the OA model [33]. The COX-2 level is increased greatly by IL-1 $\beta$ compared with healthy knee joints. For OA treatment, COX-2 inhibitor drugs has become the most commonly prescribed treatment to relieve pain and slow the cartilage breakdown process [34]. In the COX-2 inhibition with gapmer loaded HA hydrogel experiment, COX-2 silencing effects were not apparent at day 1 after induction of inflammation using IL-1 $\beta$. This may be due to time required for cellular uptake and mRNA gene silencing process and mRNA half-life. The unassisted delivery of gapmer relies on passive diffusion process and requires time to reach an effective concentration in the cytoplasm for mRNA cleavage [35], whilst the existing COX-2 mRNA requires time for degradation as mRNA has an approximate half-life of $7.1 \mathrm{~h}[36]$. Results showed a 14-day COX-2 silencing effect despite a 5-day release profile, which can be attributed to the stability of gapmers in the cellular cytoplasm. Gapmers are flanked by locked nucleic acid modification, that give protection from endogenous enzymatic degradation, and, thus, can exert silencing for an extended period that has been shown previously to be 6-10 days [9]. In our work, the prolonged silencing effect was extended over 10 to 14 days, suggesting the hydrogel-mediated delivery was effective for prolonged gene silencing. Compared with current nonsteroidal anti-inflammatory drugs for OA treatment which are orally taken several times daily and may have gastrointestinal adverse reactions [37], the local sustained release of COX-2 gapmer may reduce drug dose frequency and minimize systemic toxicity. In this work, COX-2 was the silencing target for inhibition of inflammation. The oxidized hyaluronic acid and chitosan solutions have low viscosity, which should not present a problem as injectables for future in vivo studies. It is, however, not 
possible to inject the combined HA/chitosan mix due to the fast gelation of $\sim 10$ seconds. Simultaneous injections, however, of the two solutions is possible using a two compartment syringe to allow gelation in situ. A higher number of chondrocytes have been used $(10-130$ million cells $/ \mathrm{ml})$ in the literature for in vitro tissue regeneration applications [38] than used in our experiment $\left(1 \times 10^{5}\right.$ cells/well), however, the actual chondrocyte number in cartilage is $\sim 14000$ cells $/ \mathrm{mm}^{3}$ [39], which equates to $\sim 14$ million cells $/ \mathrm{ml}$. The demonstrated CD44-mediated engagement with the hydrogel in this work suggests potential recruitment of chondrocytes that potentiates therapeutic delivery into cells. Moreover, we do not anticipate that increasing the dose of gapmers would be a problem. The mechanism driven by electrostatic incorporation into the hydrogel should allow higher levels of gapmer to be incorporated due to excess polycationic chitosan compared to the polyanionic gapmers used.

Other gapmers targeting different targets such as aggrecan degradation protease ADAMTS5 (A disintegrin and metalloproteinase with thrombospondin motifs 5) can intervene in the cartilage breakdown pathway [40] and are a current focus of our work.

In this study, we introduce a novel HA-hydrogel containing gapmer antisense oligonuclotides that has a dual modality of chondrocyte binding and gene silencing. A 5-day sustained release of COX-2 specific gapmers resulted into a 14-day COX-2 silencing effect in OA chondrocytes. The combined properties of chondrocyte binding, release and unassisted cellular entry of target-specific gapmers promotes this delivery platform for a wide panel of targets that are key regulators in OA.

\section{Acknowledgements}

The authors thank Jan Mollenhauer (Lundbeckfonden Center of Excellence NanoCAN, Institute of Molecular Medicine, University of Southern Denmark) for providing CD44-expressing and CD44-negative MCF-7 cells. This project has received funding from the European Union's Horizon 2020 research and innovation programme under Marie Sklodowska-Curie grant agreement No 642414. Laura B. Creemers is supported by the Dutch Arthritis Foundation (LLP12). 


\section{References}

[1] K. Brandt, M. Doherty, L. Lohmander, Composition and structure of articular cartilage, Osteoarthritis. New York: Oxford University Press Inc, (1998) 110-111.

[2] M.B. Goldring, The role of the chondrocyte in osteoarthritis, Arthritis Rheum., 43 (2000) 1916-1926.

[3] F. Berenbaum, F. Eymard, X. Houard, Osteoarthritis, inflammation and obesity, Curr. Opin. Rheumatol., 25 (2013) 114-118.

[4] M.B. Goldring, M. Otero, Inflammation in osteoarthritis, Curr. Opin. Rheumatol., 23 (2011) 471.

[5] J.P. Raynauld, C. Buckland-Wright, R. Ward, D. Choquette, B. Haraoui, J. Martel-Pelletier, I. Uthman, V. Khy, J.L. Tremblay, C. Bertrand, Safety and efficacy of long-term intraarticular steroid injections in osteoarthritis of the knee: A randomized, double-blind, placebo-controlled trial, Arthritis Rheuma., 48 (2003) 370-377.

[6] L. Dai, X. Zhang, X. Hu, Q. Liu, Z. Man, H. Huang, Q. Meng, C. Zhou, Y. Ao, Silencing of miR-101 Prevents Cartilage Degradation by Regulating Extracellular Matrix-related Genes in a Rat Model of Osteoarthritis, Mol. Ther., 23 (2015) 1331-1340.

[7] K.A. Howard, Delivery of RNA interference therapeutics using polycation-based nanoparticles, Adv. Drug Del. Rev., 61 (2009) 710-720.

[8] C. Wahlestedt, P. Salmi, L. Good, J. Kela, T. Johnsson, T. Hökfelt, C. Broberger, F. Porreca, J. Lai, K. Ren, Potent and nontoxic antisense oligonucleotides containing locked nucleic acids, P. Natl. Acad. Sci. Usa, 97 (2000) 5633-5638.

[9] H.S. Soifer, T. Koch, J. Lai, B. Hansen, A. Hoeg, H. Oerum, C. Stein, Silencing of gene expression by gymnotic delivery of antisense oligonucleotides, Functional Genomics: Methods and Protocols, (2012) 333346.

[10] N. Souleimanian, G.F. Deleavey, H. Soifer, S. Wang, K. Tiemann, M.J. Damha, C.A. Stein, Antisense 2'-deoxy, 2'-fluroarabino nucleic acids (2' F-ANAs) oligonucleotides: in vitro gymnotic silencers of gene expression whose potency is enhanced by fatty acids, Molecular Therapy-Nucl. Acids, 1 (2012) e43.

[11] R. Barbucci, S. Lamponi, A. Borzacchiello, L. Ambrosio, M. Fini, P. Torricelli, R. Giardino, Hyaluronic acid hydrogel in the treatment of osteoarthritis, Biomaterials, 23 (2002) 4503-4513.

[12] T.N. Snyder, K. Madhavan, M. Intrator, R.C. Dregalla, D. Park, A fibrin/hyaluronic acid hydrogel for the delivery of mesenchymal stem cells and potential for articular cartilage repair, J. Biol. Eng., 8 (2014) 1. [13] H. Tan, C.R. Chu, K.A. Payne, K.G. Marra, Injectable in situ forming biodegradable chitosanhyaluronic acid based hydrogels for cartilage tissue engineering, Biomaterials, 30 (2009) 2499-2506. [14] A. Aruffo, I. Stamenkovic, M. Melnick, C.B. Underhill, B. Seed, CD44 is the principal cell surface receptor for hyaluronate, Cell, 61 (1990) 1303-1313.

[15] R.J. Peach, D. Hollenbaugh, I. Stamenkovic, A. Aruffo, Identification of hyaluronic acid binding sites in the extracellular domain of CD44, J. Cell Biol., 122 (1993) 257-264.

[16] O. Ishida, Y. Tanaka, I. Morimoto, M. Takigawa, S. Eto, Chondrocytes are regulated by cellular adhesion through CD44 and hyaluronic acid pathway, J. Bone Miner. Res., 12 (1997) 1657-1663.

[17] M.F. Ebbesen, M.T. Olesen, M.C. Gjelstrup, M.M. Pakula, E.K. Larsen, I.M. Hansen, P.L. Hansen, J. Mollenhauer, B.M. Malle, K.A. Howard, Tunable CD44-specific cellular retargeting with hyaluronic acid nanoshells, Pharma. Res., 32 (2015) 1462-1474.

[18] K.H. Bouhadir, D.S. Hausman, D.J. Mooney, Synthesis of cross-linked poly (aldehyde guluronate) hydrogels, Polymer, 40 (1999) 3575-3584.

[19] S. Nair, N. Remya, S. Remya, P.D. Nair, A biodegradable in situ injectable hydrogel based on chitosan and oxidized hyaluronic acid for tissue engineering applications, Carbohyd. Polym., 85 (2011) 838-844.

[20] C. Jarry, J.-C. Leroux, J. Haeck, C. Chaput, Irradiating or Autoclaving Chitosan/Polyol Solutions: Effect on Thermogelling Chitosan-. BETA.-glycerophosphate Systems, Chem. Pharm. Bull., 50 (2002) 1335-1340. [21] R.D. Altman, A. Manjoo, A. Fierlinger, F. Niazi, M. Nicholls, The mechanism of action for hyaluronic acid treatment in the osteoarthritic knee: a systematic review, BMC Musculoskelet. Disord., 16 (2015) 1. 
[22] R.R. Bannuru, E.E. Vaysbrot, M.C. Sullivan, T.E. McAlindon, Relative efficacy of hyaluronic acid in comparison with NSAIDs for knee osteoarthritis: a systematic review and meta-analysis, in: Semin. Arthritis Rheum., Elsevier, 2014, pp. 593-599.

[23] H. Park, B. Choi, J. Hu, M. Lee, Injectable chitosan hyaluronic acid hydrogels for cartilage tissue engineering, Acta Biomater., 9 (2013) 4779-4786.

[24] I.E. Erickson, A.H. Huang, S. Sengupta, S. Kestle, J.A. Burdick, R.L. Mauck, Macromer density influences mesenchymal stem cell chondrogenesis and maturation in photocrosslinked hyaluronic acid hydrogels, Osteoarthr. \& Cartilage, 17 (2009) 1639-1648.

[25] L.W. Moreland, Intra-articular hyaluronan (hyaluronic acid) and hylans for the treatment of osteoarthritis: mechanisms of action, Arthritis Res. Ther., 5 (2003) 1.

[26] G. Camci-Unal, H. Aubin, A.F. Ahari, H. Bae, J.W. Nichol, A. Khademhosseini, Surface-modified hyaluronic acid hydrogels to capture endothelial progenitor cells, Soft matter, 6 (2010) 5120-5126.

[27] O. Nagano, H. Saya, Mechanism and biological significance of CD44 cleavage, Cancer Sci., 95 (2004) 930-935.

[28] I. Okamoto, Y. Kawano, H. Tsuiki, J.-i. Sasaki, M. Nakao, M. Matsumoto, M. Suga, M. Ando, M.

Nakajima, H. Saya, CD44 cleavage induced by a membrane-associated metalloprotease plays a critical role in tumor cell migration, Oncogene, 18 (1999) 1435-1446.

[29] S. Banerji, A.J. Wright, M. Noble, D.J. Mahoney, I.D. Campbell, A.J. Day, D.G. Jackson, Structures of the Cd44-hyaluronan complex provide insight into a fundamental carbohydrate-protein interaction, Nat.

Struct. Mol. Biol., 14 (2007) 234-239.

[30] K.S. Kim, S. Kim, S. Beack, J.-A. Yang, S.H. Yun, S.K. Hahn, In vivo real-time confocal microscopy for target-specific delivery of hyaluronic acid-quantum dot conjugates, Nanomed. Nanotechnol. Biol. Med., 8 (2012) 1070-1073.

[31] T.R. Hoare, D.S. Kohane, Hydrogels in drug delivery: progress and challenges, Polymer, 49 (2008) 1993-2007.

[32] J. Patterson, R. Siew, S.W. Herring, A.S. Lin, R. Guldberg, P.S. Stayton, Hyaluronic acid hydrogels with controlled degradation properties for oriented bone regeneration, Biomaterials, 31 (2010) 6772-6781.

[33] E. Hedbom, H. Häuselmann, Molecular aspects of pathogenesis in osteoarthritis: the role of inflammation, Cellular and Molecular Life Sciences CMLS, 59 (2002) 45-53.

[34] R. Bannuru, J. Wong, D. Kent, C. Schmid, T. McAlindon, Intra-articular corticosteroids may be a costeffective strategy for short-term management of knee osteoarthritis, Osteoarthr. \& Cartilage, 23 (2015)

A392-A394.

[35] C. Stein, J.B. Hansen, J. Lai, S. Wu, A. Voskresenskiy, A. Høg, J. Worm, M. Hedtjärn, N.

Souleimanian, P. Miller, Efficient gene silencing by delivery of locked nucleic acid antisense oligonucleotides, unassisted by transfection reagents, Nucleic Acids Res., 38 (2010) e3-e3.

[36] L.V. Sharova, A.A. Sharov, T. Nedorezov, Y. Piao, N. Shaik, M.S. Ko, Database for mRNA half-life of 19977 genes obtained by DNA microarray analysis of pluripotent and differentiating mouse embryonic stem cells, DNA Res., 16 (2009) 45-58.

[37] R. Chou, M.S. McDonagh, E. Nakamoto, J. Griffin, Analgesics for Osteoarthritis, (2011).

[38] R. Mauck, C.-B. Wang, E. Oswald, G. Ateshian, C. Hung, The role of cell seeding density and nutrient supply for articular cartilage tissue engineering with deformational loading, Osteoarthr. \& cartilage, 11 (2003) 879-890.

[39] R. Stockwell, The interrelationship of cell density and cartilage thickness in mammalian articular cartilage, J. Anat., 109 (1971) 411.

[40] S.S. Glasson, R. Askew, B. Sheppard, B. Carito, T. Blanchet, H.-L. Ma, C.R. Flannery, D. Peluso, K. Kanki, Z. Yang, Deletion of active ADAMTS5 prevents cartilage degradation in a murine model of osteoarthritis, Nature, 434 (2005) 644-648. 


\section{Supplementary Figures}

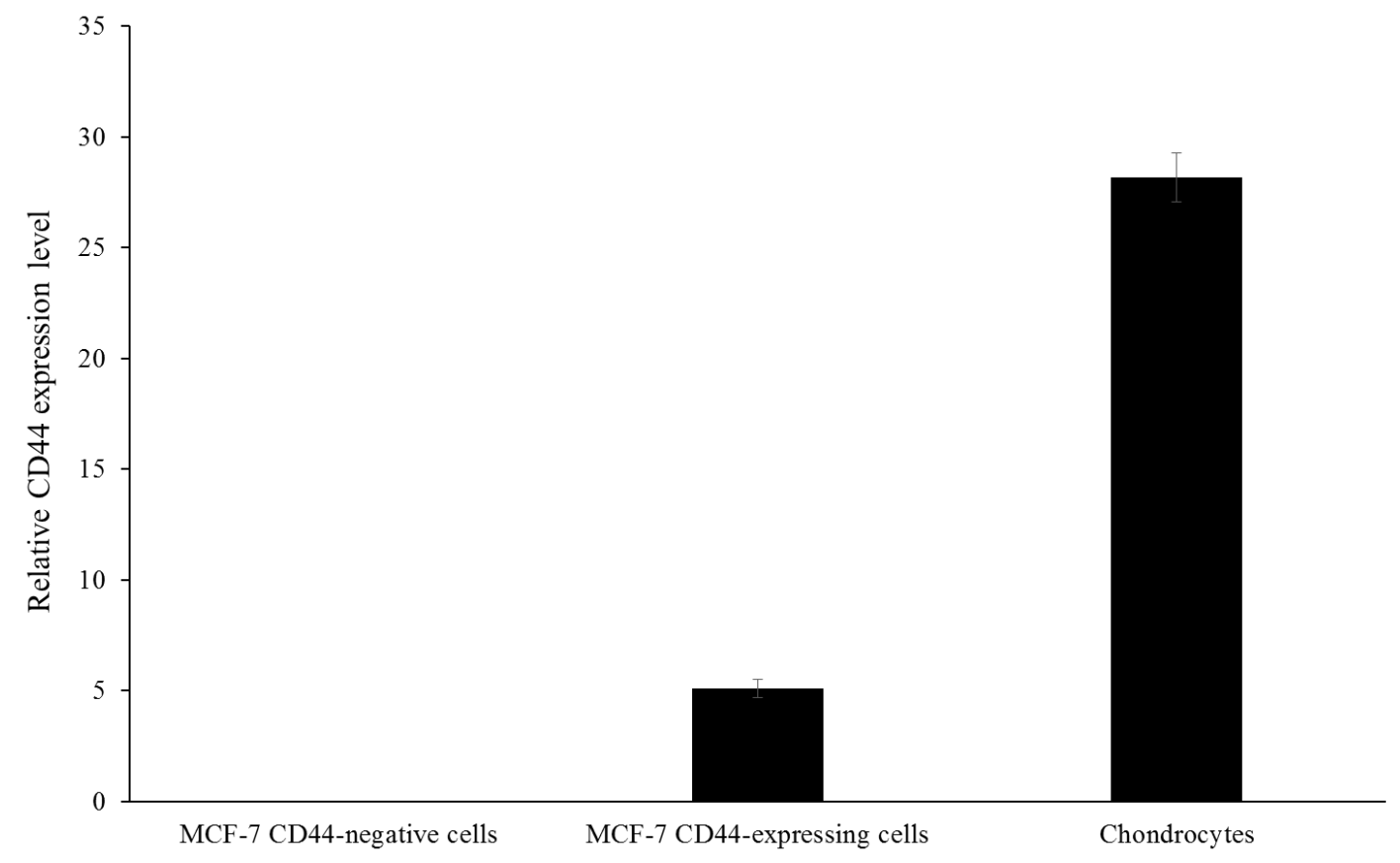

Figure 1. Determination of CD44 protein expression level on various cell types. Chondrocytes,

CD44-expressing (CD44+) and CD44 negative (CD44-) MCF-7 cells were trypsinized and suspended in block buffer. FITC-CD44 human antibody was added into cell suspensions for 30 min incubation and fluorescence intensity was measured as CD44 expression level using flow cytometry. Flow cytometer used a laser wavelength of $488 \mathrm{~nm}$ and gate events of $10000(\mathrm{~N}=3)$. 


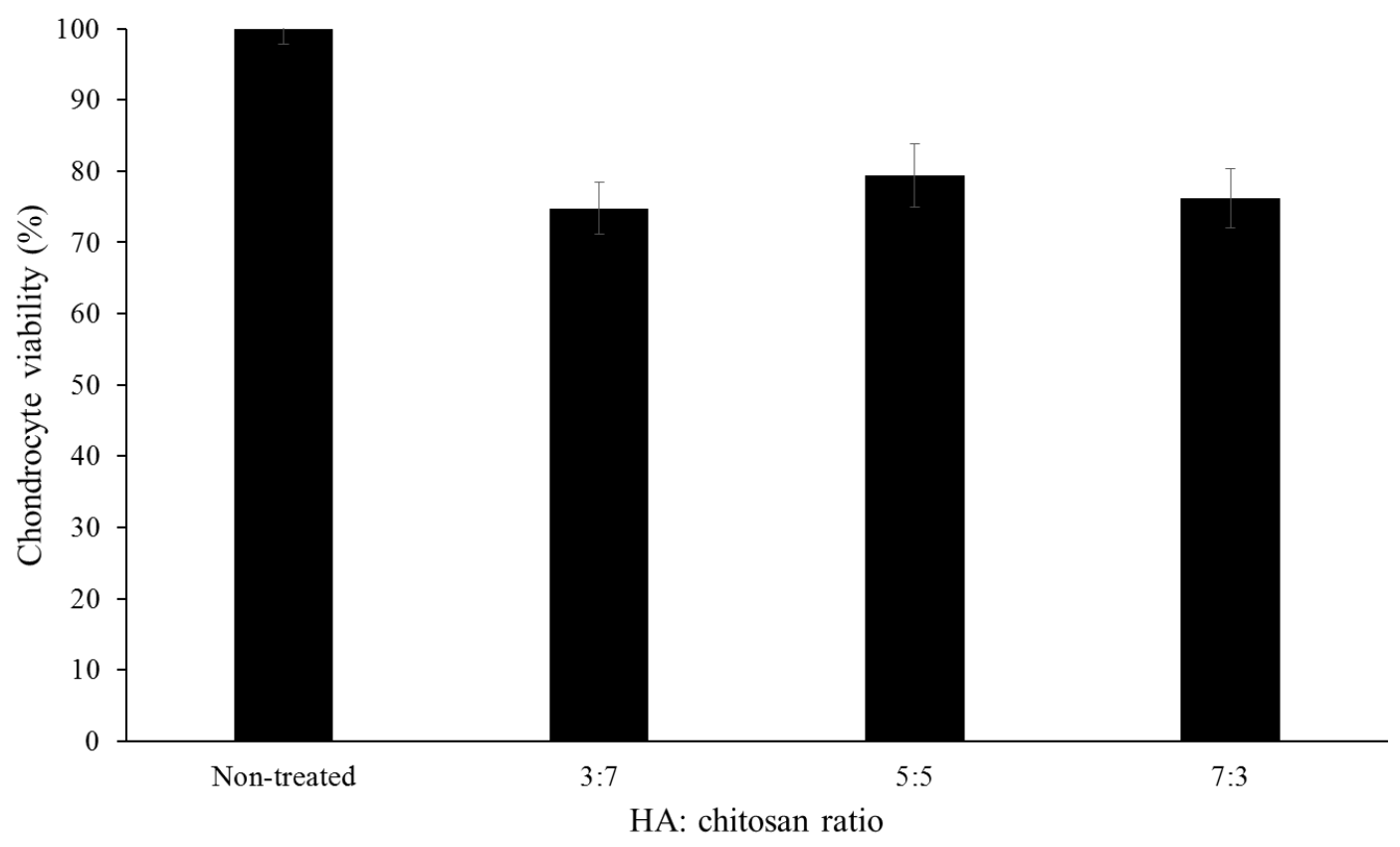

Figure 2. Viability of OA chondrocytes binding to hydrogels of different HA: chitosan ratio using an MTT assay. $5 \times 10^{4}$ cells were seeded on hydrogels of different HA:chitosan ratios (3:7, 5:5 and 7:3) in $0.5 \mathrm{ml}$ culture media. Total binding chondrocytes were collected after $36 \mathrm{~h}$ incubation and counted by NucleoCounter ${ }^{\circledR} N C$-200. Live cell numbers were determined using an MTT assay with a calibration curve from non-treated cells. Viabilities were calculated as live cells/total cells $(N=3)$. 


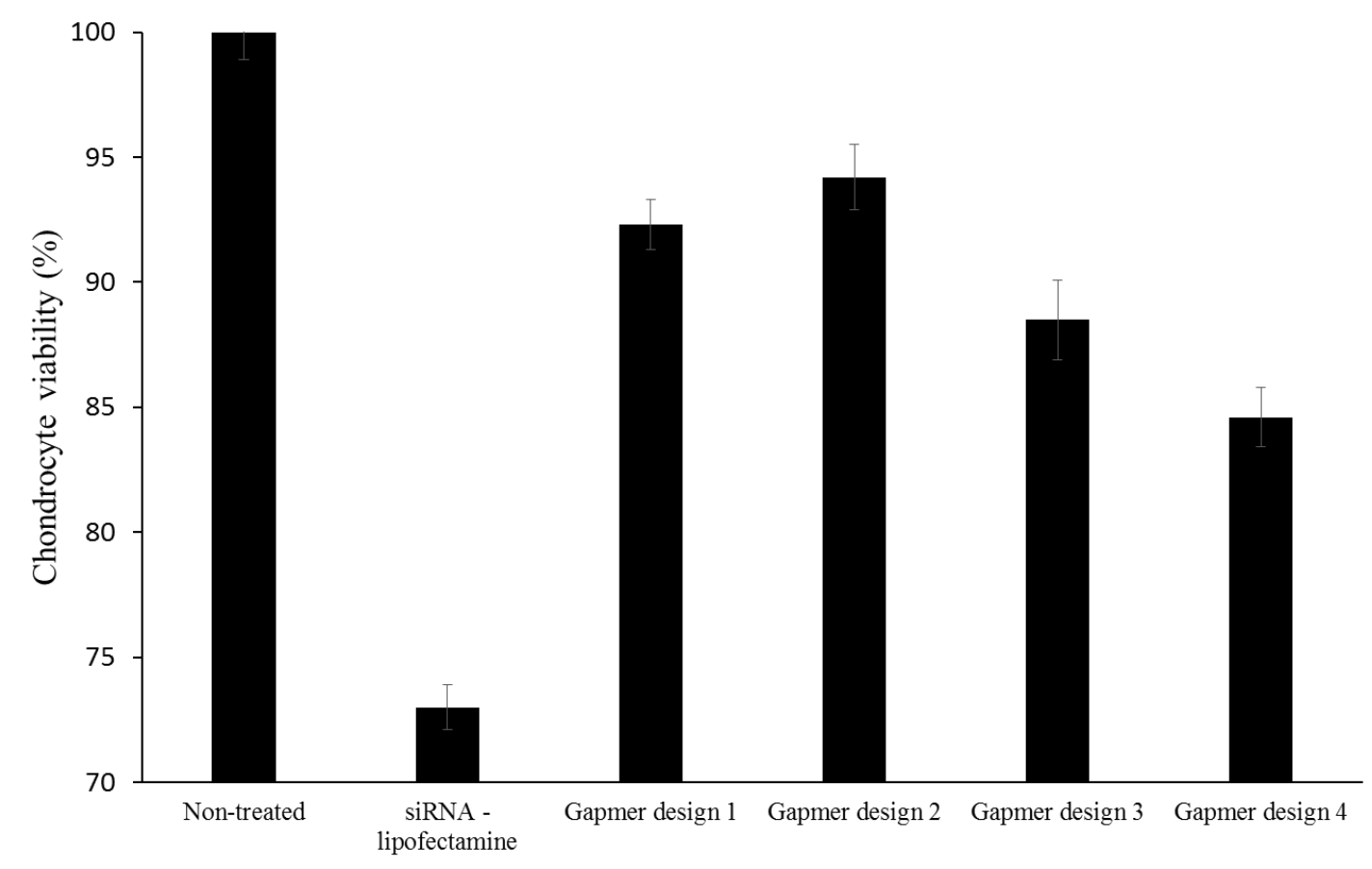

Figure 3. Viability of $O A$ chondrocytes treated with $\mathrm{COX}-2$ gapmers. Chondrocytes were treated with $500 \mathrm{nM}$ gapmers of different designs, and the viability evaluated after $24 \mathrm{~h}$ by an MTT assay with absorbance measured at $570 \mathrm{~nm}(N=3) .50 \mathrm{nM}$ siRNA with $4 \mu$ l lipofectamine was used as a control group. Gapmer design 1: 5'-lGlGlAlT dGdCdCdAdGdTdGdAdTdA lGlAlGlG-3', Gapmer 2 design: 5'-lGlGlAlT dGdCdCdAdGdTdGdA ITlAlGlA-3', Gapmer 3 design: 5'-lGlGlAaT dGdCdCdAdGdTdGdA aTlAlGlA-3', Gapmer design 4: 5'-lGlGlA dAdAdCdAdTdCdGdAdC lAlGlT-3'. ( - locked nucleic acid, $d$-deoxyribonucleotide, a palmitoylated amino locked nucleic acid). COX-2 siRNA-sense strand: 5'CAUUCCCUUCCUUCGAAAUdTdT-3' and antisense strand: 5'AUUUCGAAGGAAGGGAAUGdTdT-3'. 


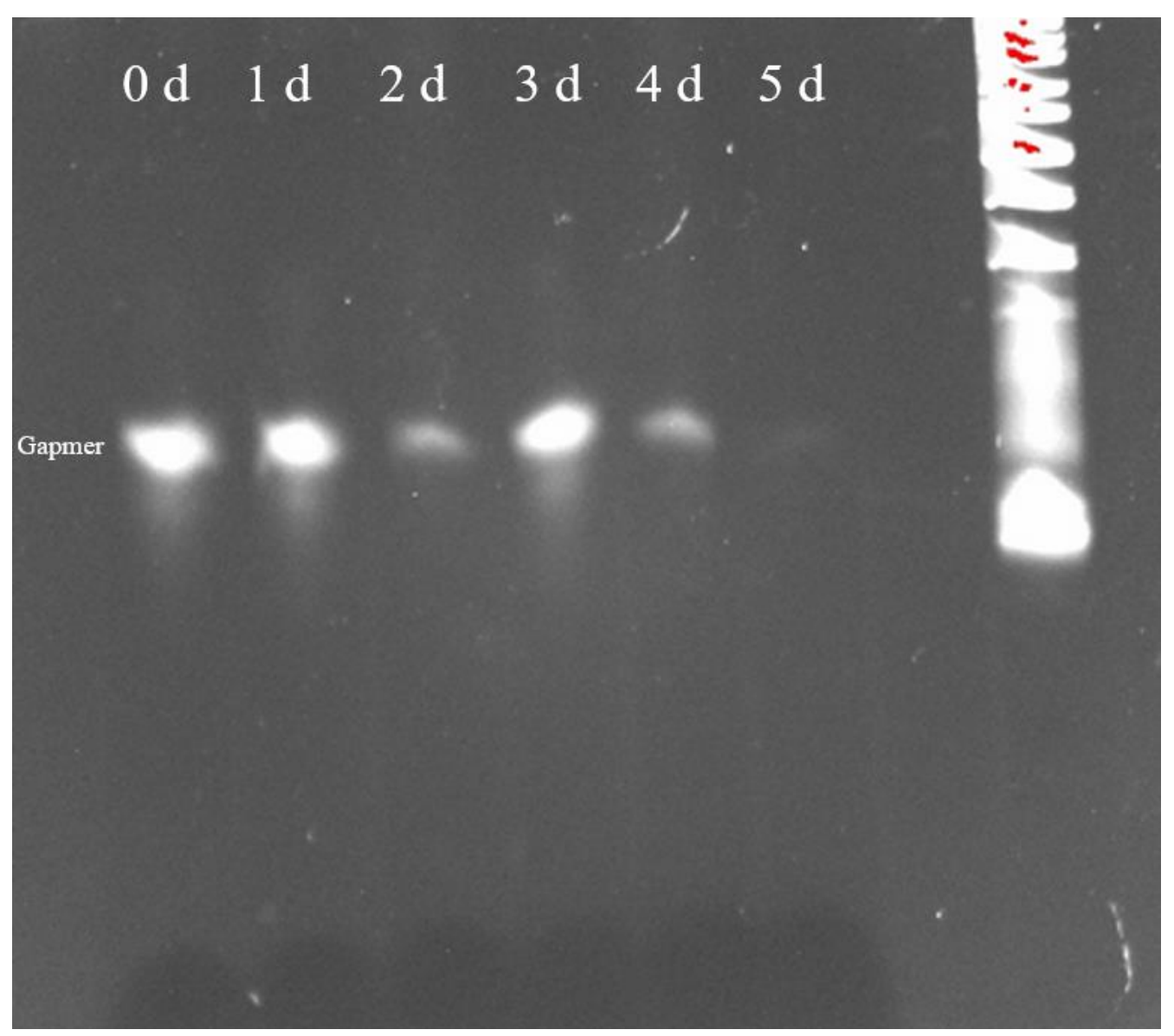

Figure 4. Gapmer structural integrity study using gel electrophoresis. Gapmer design 1 (4 nmol) was incorporated into $2 \mathrm{ml}$ hydrogel of 7:3 HA/chitosan ratio. The hydrogel was added into a 24-well plate and $0.5 \mathrm{ml}$ PBS was used as release medium. Released gapmers were loaded on a $20 \%$ polyacrylamide gel and stained with SYBR gold. The molecular weights of gapmers were compared with GeneRuler Ultra Low Range DNA Ladder (ThermoFisher). 